\title{
Differences and similarities in information seeking: children and adults as Web users
}

\author{
Dania Bilal *, Joe Kirby \\ School of Information Sciences, University of Tennessee-Knoxville, 804 Volunteer Blvd., Knoxville, TN 37996, USA
}

Accepted 25 October 2001

\begin{abstract}
This study examined the success and information seeking behaviors of seventh-grade science students and graduate students in information science in using Yahooligans! Web search engine/directory. It investigated these users' cognitive, affective, and physical behaviors as they sought the answer for a factfinding task. It analyzed and compared the overall patterns of children's and graduate students' Web activities, including searching moves, browsing moves, backtracking moves, looping moves, screen scrolling, target location and deviation moves, and the time they took to complete the task. The authors applied Bilal's Web Traversal Measure to quantify these users' effectiveness, efficiency, and quality of moves they made. Results were based on 14 children's Web sessions and nine graduate students' sessions. Both groups' Web activities were captured online using Lotus ScreenCam, a software package that records and replays online activities in Web browsers. Children's affective states were captured via exit interviews. Graduate students' affective states were extracted from the journal writings they kept during the traversal process. The study findings reveal that $89 \%$ of the graduate students found the correct answer to the search task as opposed to $50 \%$ of the children. Based on the Measure, graduate students' weighted effectiveness, efficiency, and quality of the Web moves they made were much higher than those of the children. Regardless of success and weighted scores, however, similarities and differences in information seeking were found between the two groups. Yahooligans! poor structure of keyword searching was a major factor that contributed to the "breakdowns" children and graduate students experienced. Unlike children, graduate students were able to recover from "breakdowns" quickly and effectively. Three main factors influenced these users' performance: ability to recover from "breakdowns", navigational style, and focus on task. Children and graduate students made recommendations for improving Yahooligans! interface design. Implications for Web user training and system design improvements are made. (c) 2002 Elsevier Science Ltd. All rights reserved.
\end{abstract}

${ }^{*}$ Corresponding author. Tel.: +1-865-974-3689.

E-mail addresses: dania@utk.edu (D. Bilal),kirby@utk.edu (J. Kirby). 


\section{Introduction}

The nature of the Web as a hypermedia and dynamic information retrieval system has reshaped how users seek, retrieve, and evaluate information. Use of the Web has proliferated in school and other types of libraries, but little is known about how young people and adults find information on the Web and, specifically, how these two user groups seek information in the same Web search engines. Little is also known about how well the interface design of Web search engines supports user information seeking.

Research on children's use of the Internet/Web (Bilal, 1998, 1999, 2000, 2001; Bilal \& Watson, 1998; Kafai \& Bates, 1997; Large, Beheshti, \& Moukad, 1999) and adult use of the Internet/Web (Jansen, Spink, \& Saracevic, 2000; Meghabghab, 1995; Nahl \& Meer, 1997; Palmquist \& Kim, 2000; Saracevic, 1997; Wang, Hawk, \& Tenopir, 2000; Wolfram \& Ross, 2000) shows that both user groups have cognitive difficulties constructing effective search queries, and that most of these users do not use the Web effectively.

To date, Web research has mainly focused on how children use search engines that are designed for adults (Large \& Beheshti, 2000; Large et al., 1999; Schacter, Chung, \& Dorr, 1998). No studies have investigated how adults use search engines that are designed for children.

Research that concern adults' information seeking on the Web have either included the whole Web (Catledge \& Pitkow, 1995; Lazonder, Biemans, \& Wopereis, 2000; Nahl \& Meer, 1997; Tauscher \& Greenberg, 1997; Wang et al., 2000) or a single engine such as Excite (Jansen et al., 2000; Wolfram \& Ross, 2000).

Information seeking activities may vary based on individual differences, such as age, search tasks, information retrieval systems used, and learning styles. In fact, individual differences in information seeking are of renewed interest. Recently, a special issue of the Journal of the American Society for Information Science addressed various aspects of these differences (Chen, Czerwinski, \& Macredie, 2000). However, no research in this issue examined differences in information seeking between children and adults as they use the Web.

This study examined differences and similarities in information seeking on the Web between seventh-grade children and graduate students in information science. It explored these users' thoughts (i.e., cognitive behavior), feelings (i.e., affective behavior), and actions (physical behavior) as they used Yahooligans! Web search engine/directory to find the correct answer to a fact-finding task. Although one may assume that graduate students possess higher cognitive skills than children and, therefore, ought to be better at using a search engine designed for children, the authors argue that "age" is only one interacting factor influencing information seeking. Marchionini (1995) notes, for example, that information seeking is influenced by the information seeker, task, search system, domain, setting, and search outcomes.

The main objectives of this study were to: (a) examine children and graduate students' success in finding the correct answer to a fact-finding task in Yahooligans!, (b) explore children and graduate students' information-seeking behaviors from the cognitive, physical, and affective perspectives, and (3) apply Bilal's (Bilal, 2000) Web Traversal Measure that quantifies children's and graduate students' effectiveness and efficiency in using the Web, as well as quality of Web moves they make. Results gained from this study will derive factors to use in improving the design interfaces of Yahooligans! They will also provide a framework for improving user's experience on the Web through training. 


\section{Related research}

\subsection{Children and the Web}

In a multiple-part research project, Bilal $(2001,2000)$ investigated the success and informationseeking behavior of seventh-grade science students in using the Yahooligans! search engine/ directory on three types of search tasks: fact-finding, research-based, and self-generated. Children's behavior was examined from the cognitive, physical, and affective perspectives. In part I (2000), children navigated Yahooligans! to find the correct answer to a fact-finding task about "the age of alligators in the wild and captivity" that was assigned by their science teacher. Children's behavior was captured using Lotus ScreenCam. Their affective states were elicited through exit interviews. Their prior experience in using the Web and Yahooligans! were gathered through a questionnaire that they completed at the beginning of the study. The results of the study were based on 14 usable Web sessions. The study findings reveal that $50 \%$ of the children succeeded and $50 \%$ failed. While children's cognitive behavior reflected an understanding of the search task, term relationships, search formulation, and subject hierarchies, it showed that they experienced difficulties in using the engine. Based on the Web Traversal Measure the author developed and applied, successful children were much more effective than unsuccessful ones (31.14\% vs. $12.42 \%$, respectively). This weighted score means that successful children put nearly $70 \%$ of their navigational effort toward finding the target hyperlink, whereas unsuccessful ones put nearly $88 \%$ of their effort to that end. In addition, the Measure showed a slight difference in weighted efficiency scores between successful and unsuccessful children (26.28\% vs. $22.14 \%$, respectively) and a slight difference in their quality moves scores $(32.14 \%$ vs. $28.85 \%)$. The former scores mean that children made lots of "effort" to locate the target hyperlink and complete the task. The latter scores indicate that the quality of Web moves they made did not exceed 33\%. Yahooligans! poor structure of keyword searching was a main factor that contributed to most of the "breakdowns" that the children experienced. Despite the difficulty the children had in using the engine, their high motivation surfaced as stimuli for their persistence to locate the target answer.

In part II of the research project, Bilal (2001) examined the success and cognitive and physical behaviors of seventh-grade science children in using Yahooligans! to find relevant information for an assigned research task about "the depletion of the ozone layer". Sixty-nine percent of the children partially succeeded and 31\% failed. Children's success levels were influenced by the way they approached the task. They seemed to seek specific answers to the task rather than develop understanding from the information found. Overall, children had more difficulty with the research task as opposed to the fact-finding task they performed in the previous study (Bilal, 2000). Reasons were: inadequate levels of research skills, misunderstanding of how to complete the task successfully, absence of incentive (e.g., a grade) in performing the task, and lack of engagement in the assigned topic. Children seemed to be motivated to use the Web as an end in itself rather than complete the task successfully. Like the prior study, the findings of this study revealed that Yahooligans! interface design contributed to the many difficulties that the children had experienced.

Large and Beheshti (2000) collected children's stories about using the Web to find relevant information for classroom assignments. Children perceived Web accessibility as an advantage to print sources, but found it harder to use. In a prior study, Large et al. (1999) studied the Web navigational skills of a group of middle school students as they searched for information about 
"Winter Olympics". They found that children were inefficient in using the Web and possessed inadequate navigational skills. Children were instructed to use only Infoseek and Alta Vista because they employed the same Boolean default (OR). This study examined only the "typical" moves children made in using the Web.

Schacter et al. (1998) explored the performance and searching behaviors of elementary school children in using the Web on two types of tasks: fact-finding and research. Results show that children were interactive information seekers, preferring to browse rather than plan or employ systematic and analytic search strategies. Children had difficulty finding relevant information, but were more successful in finding information on the open-ended task than the fact-finding task. The majority of children used only one Web search engine. The name of this engine is not mentioned, however. The authors examined children's analytic searches, browsing, scan-andselect, and search performance. No measure was used to examine children's efficiency, effectiveness, or quality of Web moves they made. In addition, this study did not explore children's affective states while using the Web.

In studying young adults' use of the Web, Fidel et al. (1999) observed the activities of eight high school students as they looked for information on horticulture topics for a class assignment. Results reveal that students were inefficient in using the Web. The authors did not employ a measure for examining students' efficiency.

\subsection{Adults and the Web}

Few studies explored how adult users interact with the Web. Wang et al. (2000), for example, examined the information seeking behaviors and success of 24 graduate students in information science in using Web resources to find the correct answers to two assigned factual tasks. Students' success level varied by the search task. Sixty-seven percent were successful on the first task, whereas $92 \%$ were successful on the second one. Findings also show that the students had limited understanding of how the Web worked and how search engines differed from one another. The authors did examine users' affective states, but measured effectiveness based on success, and efficiency based on the time they took to complete the tasks.

In a study of the Excite search engine, Jansen et al. (2000) examined the searching behaviors of 18,000 users based on analysis of transaction logs of a 51,000-query data set. Results show that users did not have many queries per search, rarely modified queries, and used advanced search syntax minimally in constructing queries. Fewer than $10 \%$ of the queries submitted, for example, employed Boolean operators; of these, users applied the Boolean "AND" operator incorrectly. Due to the nature of this study, the characteristics (e.g., age) of these users are unknown. Therefore, one cannot determine individual differences in information seeking among these users.

Catledge and Pitkow (1995) pioneered a study at the Georgia Institute of Technology that captured the browsing behavior of staff, faculty, and students. The study findings show that following a link and using the Back command were the most frequent Web actions these users made $(52 \%$ and $41 \%$, respectively). The authors hypothesized that users who are categorized as "browsers" spent less time on a Web page than those who are categorized as "searchers". In a follow-up study, Tauscher and Greenberg (1997) explored the History mechanism in selected Web browsers. They collected browsing data from 23 participants. They found that $58 \%$ of the pages the participants visited were re-visits, and that these pages were re-visited through activating the 
Back command. The authors contend that Web browsing is a "recurring system ... where users predominantly repeat activities they had invoked before, while still selecting new actions from the many that are possible" (p. 400). It is unclear why most users activated the Back command rather than used shortcuts, such as History list, Go list, or URL list.

Overall, the literature reveals that when examined, effectiveness in using the Web is measured by success, and that efficiency is measured by the time taken to complete tasks. Web research has overlooked using a measure of effectiveness and efficiency in using the Web that goes beyond success and time taken to complete tasks. In addition, it has neglected to study differences and similarities in information seeking on the Web between children and adults, especially in using the same search engine.

\subsection{Information seeking studies}

Information seeking studies support the notion that users exhibit common characteristics of information behaviors at different stages of the information seeking process. Kuhlthau (1993) found young adults' and adults' information seeking evolved in six stages that were associated with cognitive, physical, and affective states. Although her study was conducted in a traditional library environment, Kuhlthau's findings suggest that user cognitive, physical, and affective states are a driving force in any information seeking process.

Nahl's ACS model of information behavior is tuned to Internet users (cf. Nahl, 1997). It is composed of three elements: (A) affective, (B) cognitive, and (C) sensorimotor. Nahl maintains that to begin with, there are intentions or information needs (A), which lead one to thoughts about solutions (C), which finally eventuate in some related overt action (S). ACS is one of the earliest models to associate user affective, cognitive, and physical behaviors with information seeking on the Web.

Wilson (2000) perceives information seeking, searching, and use as associated with different stages of a goal-oriented problem-solving process. These stages are: problem recognition, problem definition, problem resolution, and solution statement (where needed). Wilson's model does not integrate the three triads of information seeking (i.e., cognitive, physical, and affective behaviors) into this process.

Marchionini's model of the information seeking process describes eight stages that develop in parallel: problem recognition, problem understanding, choosing a search system, formulating a query, executing a search, examining results, extracting information, and reflecting/iterating/ and stopping (cf. Marchionini, 1995). This model may be tuned to information seeking in electronic environments. It also relates to Kuhlthau's model of the information search process, except that it does not integrate cognitive, physical, and affective behaviors into these stages (cf. Kuhlthau, 1993).

Ellis (1989), Ellis, Cox, and Hall (1993) and Ellis and Haugan (1997) proposed a model of the information seeking process based on studies of the information behavior of researchers in the social sciences, physical sciences, and in engineering. The model describes a set of common characteristics of information seeking activities: starting, chaining, browsing, differentiating, monitoring, extracting, verifying, and ending. In Choo, Detlor, and Turnbull (1999) integrated a version of this model with Anguilar's four modes of scanning (1967) to examine user information seeking on the Web in a business environment. The authors concluded that a behavioral framework that relates motivations and Web moves "may be helpful in analysing Web-based 
information seeking" (p. 15). Motivations in this framework relate to affective states that Kuhlthau (1993) and Nahl (1997) consider as one of the main factors influencing information-seeking activities.

Qiu (1994) modeled the path patterns users followed in navigating an early hypertext system (i.e., Hyperties). The author found that Zipf's distribution was the best fit for the frequency of user node visiting. Another important finding is that the type of search task (i.e., specific vs. openended) did not influence the path patterns users followed. Qiu's study was the first attempt at developing hypertext path pattern models. Today, hypertext systems, especially the Web, have progressed exponentially in terms of use and navigational features. Yet, the study of user weighted effectiveness and efficiency in using these systems is still in its infancy.

\subsection{Theoretical framework}

In interacting with an information retrieval system, such as a Web search engine, it is important to learn the underlying cognitive processes, actions, and affective states of information seeking in order to obtain a holistic view about the user's behavior (Dervin, 1983; Ingwersen, 1982, 1992, 1996; Kuhlthau, 1993; Nahl, 1997). Presently, we have no knowledge of how these states vary among users of different maturity levels when seeking information on the Web. In addition, user success in finding desired information has been used as the main measure of user effectiveness. Similarly, user efficiency has been measured by the time a user takes to complete a specific task. Thus, in addressing young people's and adults' use of the Web, a major research effort has to be directed towards analyzing differences and similarities in information seeking between children and adults. In addition, efforts should be aimed at applying a measure that quantifies these users' effectiveness and efficiency in using the Web, as well as quality of the Web moves they make. This study is a first attempt to fill this gap in the literature.

\section{Research questions}

This study examined children's and graduate students' information seeking on the Web from three perspectives: cognitive, physical, and affective behaviors. The cognitive behavior relates to knowledge, comprehension, problem solving, and critical interpretation (Nahl, 1997). The physical behavior concerns actions made other than searching and browsing, such as screen scrolling, activating Netscape command features (e.g., Back command), target location and deviation, and time taken to complete the task. The affective behavior relates to feelings, perceptions, attitudes, and motivation.

This study sought answers to these questions:

1. How successful are children and graduate students in finding the correct answer to a fact-finding task in Yahooligans!?

2. What similarities and differences in the cognitive behaviors do children and graduate students demonstrate in using Yahooligans!?

3. What similarities and differences in the physical behaviors do children and graduate students demonstrate in using Yahooligans!? 
4. What differences in weighted traversal effectiveness, efficiency, and quality Web moves does the Web Traversal Measure reveal between children and graduate students?

5. What affective behaviors do children and graduate students experience in using Yahooligans!?

\section{Method}

This study employed both quantitative and qualitative inquiry methods. The quantitative method provides empirical data about the behavior, success, problem solving, Web navigation skills, and knowledge of using Yahooligans! This method requires that these observations be recorded and viewed at a later time. Lotus ScreenCam (http://www.lotus.com), a Windows-based software package that records and replays captured activities in Web browsers, was employed to achieve this goal.

The qualitative method generates data from interviews and journal writing and provides an understanding of the behavior data that result from the quantitative method. Children's affective states were captured via one-on-one structured interviews at the end of the study. Graduate students' affective states were extracted from the journals they kept during the search process.

\subsection{The setting}

\subsubsection{Study I. Middle school students}

This study took place at a middle school (designated Middle School for confidentiality purposes), grades 7-9, located in East Tennessee. The library at the School was the site for this experiment. Three science classes taught by one teacher were selected for this study. Prior to the research experiment, the library had two computers with an Internet connection. Three additional computers were networked and connected to the Internet to accommodate use of five computers at a time. Lotus ScreenCam was installed on each of the five computers and pre-tested for proper operation. Netscape Web browser was used and Yahooligans! was set up as the default home page in the browser. Each child's Web activities were captured, saved, and transferred electronically to the researchers' computers (Bilal, 2000).

\subsubsection{Study II. Graduate students}

This study took place in a computer laboratory at the School of Information Sciences, the University of Tennessee, Knoxville. Lotus ScreenCam was installed on 12 computers and pretested for proper operation. Netscape browser (Navigator) was used and Yahooligans! was set up as the default home page in the browser. Each graduate student's Web activities were captured, saved, and transferred electronically to the researchers' computers.

\subsection{Participants}

\subsubsection{Study I. Middle school}

The population of this study consisted of 90 seventh-grade science students taught by one science teacher. Due to the School's Internet Use Policy, children's parental consent to use the Internet was sought. Out of 90 invitations for participation, 30 consent forms were received. Of 
these, 25 children were willing to take part in this study. Three were involved in pilot testing, leaving 22 children in the sample.

\subsubsection{Study II. Graduate students}

The population in this study involved 12 graduate students in information science who were enrolled in an introductory computer course taught by the first author. Three students were absent during the research experiment, leaving nine students in the sample.

\subsection{Prior knowledge of the Web}

Children and graduate students had novice knowledge of using the Web. The Middle School offered curriculum-related user instruction that included use of the Web. This instruction covered basic search strategies in using selected search engines, such as Alta Vista. Yahooligans! was not included in this instruction.

Graduate students were introduced to the Web by the first author as part of the computer course instruction. The instruction included an overview of how to use the Web and search engines. Thus, both student groups were novices to the Web and possessed equivalent formal Web training prior to the research experiment.

\subsection{The search task}

A fact-based task was given to both groups to search in Yahooligans! Fact-based tasks are usually simple, certain, and uncomplicated in nature. Such tasks have a target answer that may be a date, a location of an address, a lifespan of an animal, and the like. As Vakkari (1999) maintains, "simple tasks are routine information processing tasks where the elements of the task are predetermined, i.e., [the user] knows them" (p. 826); meaning that the requirements of the task (e.g., the amount of information needed) can be determined by the user. In Study I (middle school students), the science teacher assigned the following fact-based task to search in Yahooligans!: How long do alligators live in the wild, and how long in captivity? In Study II (graduate students), the authors assigned the same task to the graduate students to search in Yahooligans!

\subsection{Instruments}

The researchers developed an exit interview instrument that captured children's affective states at the end of the experiment. Graduate students were given forms (journals) to use for documenting their feelings concurrently during searching. Interview and journal writings data were tabulated and analyzed.

\subsection{Measurement}

In a prior study, Bilal (2000) developed and used a Web Traversal Measure that examined children's weighted traversal effectiveness, efficiency, and quality of Web moves. In this study, the author applied this measure to compare children and graduate students' effectiveness, efficiency, and quality of their Web moves. 
The Web Traversal Measure is based on a weight that is assigned to every Web move a user makes. Traversal in this measure is defined as all moves a user makes including searching, browsing, screen scrolling, backtracking (using Netscape Back button), search looping (re-execution of searches previously made), and hyperlink looping (re-activation of hyperlinks previously visited). Effectiveness is evaluated in terms of the amount of effort a user makes to locate a target hyperlink or page. Efficiency is assessed based on the weight of relevant moves a user makes out of the total traversal moves to complete the task. Quality moves are computed by quantifying the percentage of relevant moves a user makes out of the total traversal moves (i.e., quality vs. quantity). A scoring method was applied based on all possibilities for search moves (e.g., alligator, wild, captivity) and hyperlink activation (e.g., Science and Nature) a child or a graduate student might make in Yahooligans! Due to the nature of the titles of hyperlinks (i.e., accurate, misleading, vague) and the nature of their descriptions (i.e., accurate, misleading, vague), a three-point relevance scale was used. A score of 1 was given to either a relevant search move or relevant hyperlink activation; a score of 0.5 was assigned to either a semi-relevant search move or a semi-relevant hyperlink activation; and a score of 0 was given to an irrelevant search move or hyperlink activation. Relevance as described by Bilal (2000, p. 650), was determined as follows:

$\mathbf{1}=$ Relevant, was assigned to a search or hyperlink which, based on its formulation and/or description, is appropriate or appears to lead to the desired information and it does.

Search example: alligator

Hyperlink example: Science and Oddities: Living Things: Animals

0.5 = Semi-relevant, was assigned to a search or hyperlink which, based on its formulation and/ or description, is appropriate or appears to lead to the desired information but it does not.

Search example: alligator in captivity

Hyperlink example: Wildlife

$\mathbf{0}=$ Irrelevant, was assigned to a search or hyperlink which, based on its formulation and/or description gives no indication of and does not contain information relating to the search task.

Search example: Life lines

Hyperlink example: Biology Database

To compute effectiveness, efficiency, and quality of Web moves, three equations were derived. These equations take into account the Transcribed Moves (TMs) (i.e., all traversal moves), and Selection Actions (SAs) (i.e., moves that include only searching and/or hyperlink activation). Each SA is assigned a "weight" (WSA) based on its degree of relevance. These equations are:

Weighted effectiveness score:

$$
\sum_{i=1}^{i=n}\left(\mathrm{WSA}_{i} * \mathrm{SA}_{i}\right) / \sum_{j=1}^{j=m}\left(\mathrm{TM}_{j}\right)
$$

where $n$ is the total number of SAs and $m$ is the total number of TMs to the target hyperlink. 
Weighted efficiency score:

$$
\sum_{i=1}^{i=n} \mathrm{WSA}_{i} / \sum_{j=1}^{j=k} \mathrm{TM}_{j}
$$

where $n$ is the total number of SAs and $k$ is the total number of all TMs.

Quality moves:

$$
\sum_{i=1}^{i=n} \mathrm{SA}_{i} / \sum_{j=1}^{j=k} \mathrm{TM}_{j},
$$

where $n$ is the total number of SAs and $k$ is the total number of all TMs. See Appendix A for an example of how the measure is applied.

\subsection{Success measure}

Both student groups were judged to be successful if they found and extracted the correct fact (i.e., age of alligator in the wild and in captivity). They were judged to be partially successful if they submitted an incomplete answer (i.e., age of alligator in the wild or in captivity). They were judged to be unsuccessful if they submitted an incorrect answer. The authors and a trained research assistant evaluated the search results children and graduate students submitted.

\subsection{Procedures}

Study I (middle school students) began in April 1998. The study took place in a Middle School located in East Tennessee. The School was mainly selected for its pioneering efforts in integrating the use of technology into the classroom curriculum. The research experiment took place in the School's media center. Children were taken by the authors from their science class to the School's media center five at a time. They each signed a consent form and used a computer station that had Yahooligans! as the default Web page. Children were not given instruction as to how to use Yahooligans! The intent was to examine how, as novices, these children use Yahooligans! that is specifically designed to support their information seeking. Children were encouraged to ask questions as needed. Each student was assigned 30 minutes to complete the task. When technical problems occurred, the student was given additional time to complete the task. At completion, each student's Web session was saved and transferred electronically to the researchers' computers. Each session was replayed, analyzed, transcribed, and coded by the researchers and a trained research assistant.

Study II (graduate students) started in April 1999. The nine students who expressed their willingness to participate in this study signed consent forms and proceeded with searching Yahooligans! They kept journals that documented their experiences and feelings during searching. At completion, each student's Web session was saved and transferred electronically to the researchers' computers. Each session was replayed, analyzed, transcribed, and coded by the researchers and a trained research assistant. 


\section{Limitations of the study}

The study of middle school students was limited to seventh-grade students in three science classes. It took place at one middle school and included use of only one search engine. The children who participated in this study may not represent the cognitive, physical, and affective behaviors of all middle school students in Tennessee, nor may they represent the whole population of seventh-grade science students.

The simplicity of Yahooligans! may influence graduate students' success level and information seeking behavior. Another limitation is that the graduate students in information science who participated in this study may not represent all graduate students in Tennessee, nor may they represent all graduate students in this field of study. Absence of concurrent verbal reports that would capture the dynamics of these users' Web moves and that would explain their affective behaviors is an additional limitation.

\section{Results}

The results are reported within the context of the five research questions posed. Due to loss of data, the results were based on 14 out of 22 children's Web sessions that were usable. All graduate students' sessions were usable.

1. How successful are children and graduate students in finding the correct answer to a fact-finding task in Yahooligans!?

Graduate students were more successful in finding the correct answer to the search task than children. Eighty-nine percent of the students found the correct answer as opposed to $50 \%$ of the children. Only one graduate student was "partially" successful. He/she submitted the answer as "alligators live up to 50 years in captivity". The children who failed either did not locate the target hyperlink, or when they did, they did not view the text from the target page.

2. What similarities and differences in the cognitive behaviors do children and graduate students demonstrate in using Yahooligans!?

The cognitive behavior of both student groups was observed in terms of searching and browsing moves.

Searching moves. Sixty-four percent of the children began their initial moves in Yahooligans! by performing analytic searches and $36 \%$ by browsing subject hierarchies. In contrast, $67 \%$ of the graduate students started their initial moves by browsing subject hierarchies and $33 \%$ by making analytic searches.

Children made 56 searches (77\%) using single concepts, 5 searches (7\%) using two concepts, and 11 searches $(15 \%)$ using phrases or natural language. The average number of search queries per child was $M=5.1$.

Graduate students made 2 searches (73.3\%) using single concepts, 11 searches $(13.3 \%)$ using two concepts, and 2 searches (13.3\%) using multiple concepts. The average number of search queries a graduate student made was $M=1.66$.

Browsing moves. Overall, children and graduate students activated appropriate categories and hyperlinks, and most of the subject categories and hyperlinks they browsed were identical (Animals, Science and Nature, Alligators and Crocodiles, Gator Hole, St. Augustine Alligator Farm, and The 
American Alligator). Children browsed more than searched by keyword ( $M=8.4$ vs. $M=5.1$, respectively) (Fig. 1). They browsed slightly more sites than graduate students did ( $M=8.4$ vs. $M=7.5$, respectively). In addition, children looped searches (re-executed searches previously made) and hyperlinks (re-activated hyperlinks previously visited) more often than graduate students did ( $M=5.1$ vs. $M=0.62$, respectively). It is not surprising to find that children browsed more sites and made more analytic searches than did graduate students, especially since they took more time to complete the task. In addition, children seemed to be less focused on the task than were graduate students as was evident in the mean scores of looping activities. What may also have contributed to this high variance in looping is children's lower cognitive recall (Siegler, 1991). Given the fact that the Web imposes memory overload that reduces recall during navigation (Cockburn \& Jones, 1996), children become prone to loop searches and hyperlinks more frequently than adults.

3. What similarities and differences in the physical behaviors do children and graduate students demonstrate in using Yahooligans!?

The physical behavior was examined in relation to the moves children and graduate students made other than searching and browsing. These included backtracking (use of Netscape Back button), screen scrolling, target location and deviation, and the time taken to complete the task.

Backtracking. Fig. 1 shows that children backtracked twice as often as graduate students ( $M=6.1$ and $M=3.1$, respectively). Neither children nor graduate students who backtracked used shortcuts to navigate among Web pages (e.g., the Netscape Go button, History list, or URL list) when they were far away from the pages they previously visited. This finding may indicate

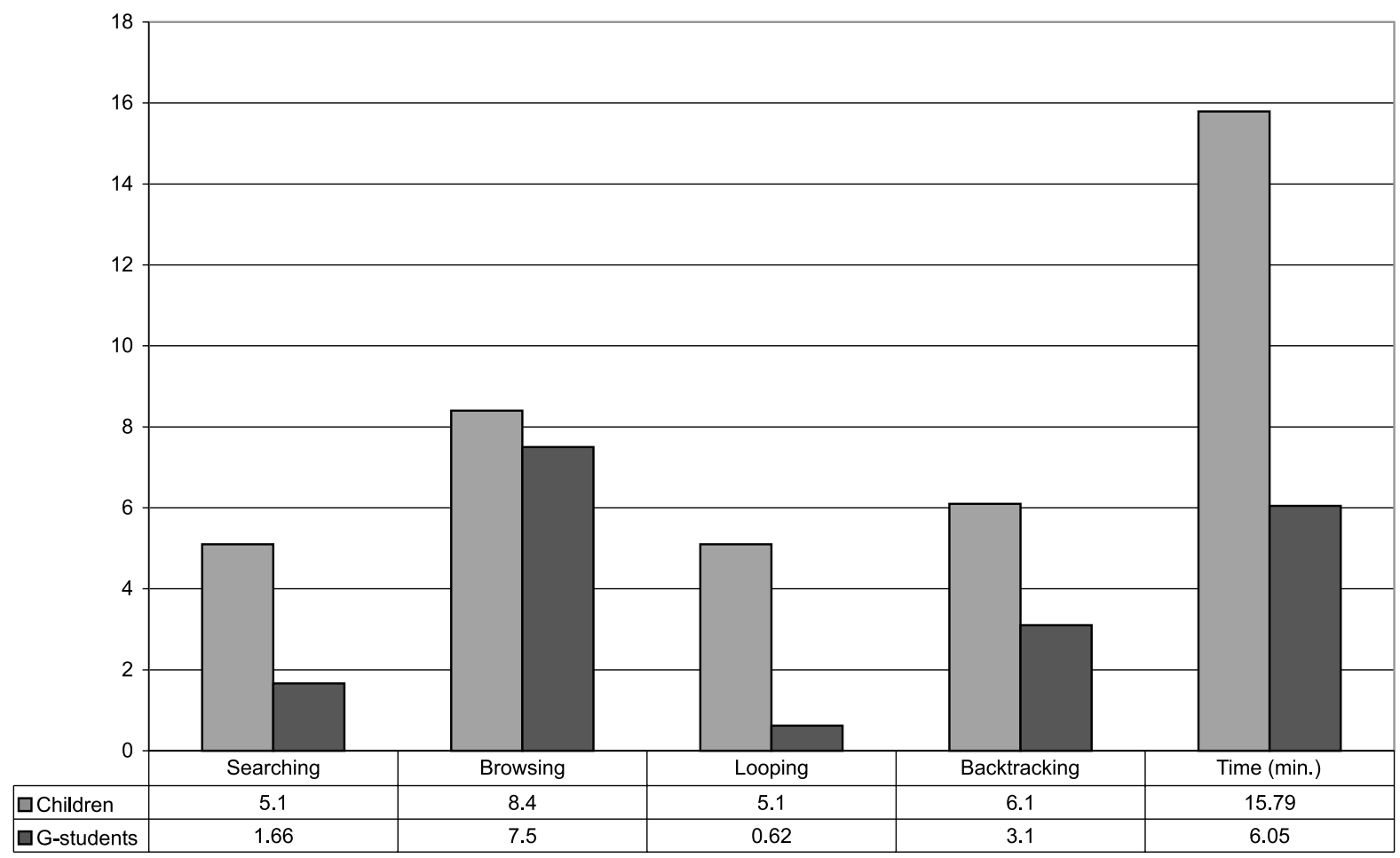

Fig. 1. Children's and graduate students' Web activities by mean. 
that neither group was familiar with these Netscape command features, or that they preferred using this command to revisit previous Web pages. The difference in backtracking moves between the two student groups may be explained by the fact that children browsed more hyperlinks, made more analytic searches, retrieved more pages and, subsequently, backtracked multiple times to revisit previous pages.

Screen scrolling. Screen scrolling was examined to determine the percentage of results both student groups viewed. Screen scrolling was not accounted for when the results were displayed in short screens. Children scrolled the results less often than graduate students did (69\% vs. 91\%, respectively).

Target location and deviation. Three targets were identified: target Web site, target hyperlink, and target home page. Activating the target hyperlink from the target site was considered as a target location. Extracting the answer from the target home page was judged as location of the target answer. Leaving the target home page without marking or printing the answer was judged as target deviation. Children and graduate students demonstrated different behaviors. The mean number of Web moves children made to complete the task was $M=49$ as opposed to $M=20$ by graduate students. Three children deviated from the targets when they were in the correct Web space. Four other children never activated the target hyperlink, even though they were in the correct Web space. In contrast, all graduate students, except for one, located the three targets and did not deviate from them. Graduate students were more thorough and "systematic" in their navigation than children were.

Traversal time. Traversal time was calculated from task initiation to completion. Children took more time to complete the task than did graduate students $(M=15.79 \mathrm{~min}$ vs. $M=6.05$, respectively) (Fig. 1). Graduate students were more efficient in completing the task than were the children. What may explain this efficiency is graduate students' ability to recover quickly from "breakdowns" caused by keyword searching by browsing subject hierarchies. On the contrary, most children persisted in searching by keyword even when their searches, including those that were re-executed, returned zero hits. They were unable to recover effectively from the "breakdowns" they experienced.

In sum, the findings of the cognitive and physical behaviors reveal that, regardless of children's and graduate students' success levels, similarities and differences in their information seeking were found.

Similarities in information seeking.

Children and graduate students:

- Employed keyword searching.

- Were more successful when they browsed subject hierarchies than when they searched by keyword.

- Looped searches and hyperlinks.

- Activated Netscape Back command exclusively to navigate among the Web pages they retrieved.

- Did not use any navigational shortcuts (e.g., History list, Go list, Home, Bookmarks).

- Had inadequate knowledge of how to use Yahooligans!

- Encountered difficulties in finding relevant hits.

- Did not quit using the engine, despite the difficulties most of them encountered in finding the target information. 
Differences in information seeking.

There were more differences than similarities in information seeking between children and graduate students. These differences are summarized below:

- Graduate students employed advanced search syntax. No child used this syntax.

- Graduate students browsed more than searched by keyword. Children browsed and search nearly equally.

- Graduate students scrolled the returned results more often than children did.

- Graduate students made fewer Web moves than children did to complete the task.

- Graduate students looped searches and hyperlinks much less often than children did.

- Graduate students backtracked much less often than children did.

- Graduate students did not deviate from any designated target whereas most children did.

- Graduate students were able to recover quickly from "breakdowns" caused by keyword searching, whereas children were not.

- Graduate students adopted a "linear or systematic" browsing style in locating the information, whereas most children had a "loopy" style.

- Graduate students took less than half the time children took to complete the task.

- Graduate students' "weighted" effectiveness, efficiency, and quality of Web moves were higher than those of the children's (Fig. 2).

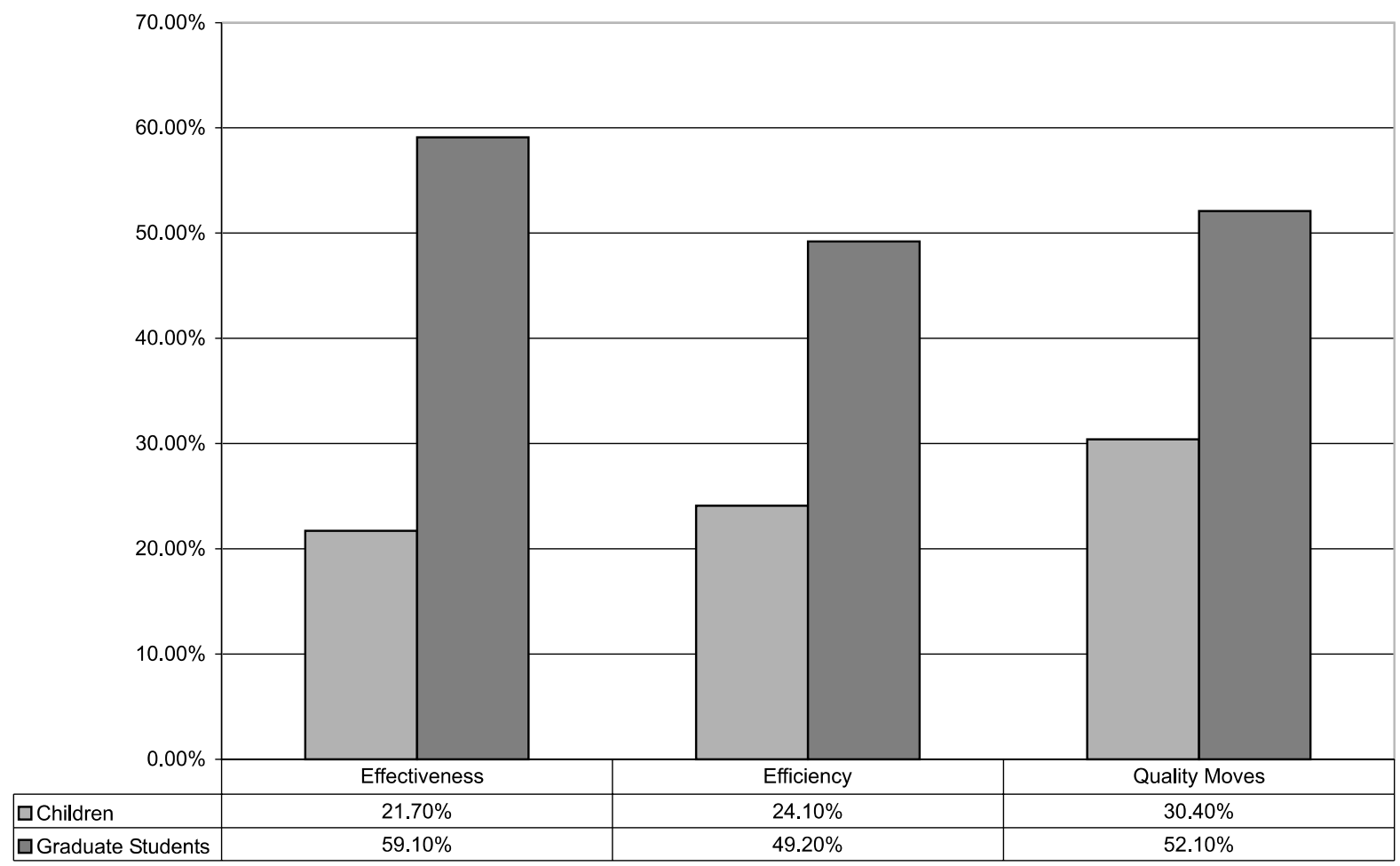

Fig. 2. Children's and graduate student's weighted Web traversal scores. 
4. What differences in weighted traversal effectiveness, efficiency, and quality Web moves does the Web Traversal Measure reveal between children and graduate students?

Children's and graduate students' weighted traversal effectiveness, efficiency, and quality of Web moves were computed based on Bilal's Web Traversal Measure (cf. Bilal, 2000). As shown in Fig. 2, children's weighted effectiveness, efficiency, and quality of Web moves were much lower than those of graduate students. Children's weighted effectiveness score was $21.7 \%$ as opposed to $59.1 \%$ by graduate students. These scores mean that children put nearly $78 \%$ of their effort toward locating the target hyperlink, whereas graduate students put nearly $40 \%$ to that end. Children's weighted efficiency score was $24.1 \%$ as opposed to $49.2 \%$ by graduate students. These scores indicate that nearly $25 \%$ of the moves children made were either relevant or semi-relevant, whereas nearly $50 \%$ of the moves graduate students made were semi-relevant or relevant. In addition, the quality of children's Web moves was $30.4 \%$ as opposed to $52.1 \%$ by graduate students. These scores denote that out of all the moves children made to complete the task nearly $31 \%$ were quality moves, whereas out of all the moves graduate students made nearly $53 \%$ were quality moves. Consequently, graduate students outperformed children in traversing Yahooligans!

5. What affective states do children and graduate students experience in using Yahooligans!?

Children's affective states. Most children (85\%) were motivated to use the Web. Reasons were: ability to search by keyword, self-confidence, challenge, convenience, ease of use, and discovery. Some comments were: "[Yahooligans!] showed me I could do it because I didn't know I could do it; I know I can do it ... [it is] a challenge to find pictures". Although these children enjoyed using Yahooligans! and the Web, 43\% were confused and frustrated. Reasons mentioned were difficulties finding relevant results, confusing screen display, and slow response time. The positive feelings the children experienced, however, were stimuli for their persistence in using the engine. No child quit searching before his/her Web session allotted time ended.

Graduate students' affective states. Eighty-nine percent of the graduate students expressed satisfaction and comfort after they completed the task and found the answer. Forty-five percent were satisfied, comfortable, and felt at ease during searching. Over 50\% were frustrated during searching. Reasons were lack of matches with Boolean searches, task difficulty, and the time it took to find the answer. One student commented, for example, "I was annoyed because I was unable to determine from the help files how Yahooligans! uses or supports Boolean searches".

Obviously, the frustration children and graduate students experienced was caused by their inability to find matches, difficulty finding the correct answer to the task, inadequate knowledge of how to use the engine, poor structure of the so called "keyword searching" and inadequacy of Yahooligans! interfaces.

\section{Discussion}

This study examined the success and cognitive, physical, and affective behaviors of children and graduate students in using the Yahooligans! Web search engine/directory to find the correct answer to a fact-finding task. Despite the fact that graduate students outperformed children, similarities and differences in information seeking were found between the two groups. This finding suggests that "age" was not a factor that influenced information-seeking behavior. This is especially true for children since they used a search engine that is specifically designed for their age 
level. The factors that seem to influence information seeking were ability to recover from breakdowns, navigational style, and focus on task.

The fact that $50 \%$ of the children failed to find the correct answer to a fact-finding task in a search engine/directory designed specifically for their age level raises an issue about their ability to use search engines that are designed for adults. Researchers, teachers, and information professionals need to address this issue when dealing with children's use of the Web.

This study revealed that, overall, both student groups were unsuccessful when they searched by keyword and that they were more successful when they browsed subject hierarchies. This finding indicates that in using Yahooligans!, "Web searchers" may be less successful than "Web browsers" mainly because the engine does not index its Web pages as thoroughly as other Web search engines do.

Children and graduate students had inadequate knowledge of how to use Yahooligans! Since graduate students were familiar with advanced search syntax (Boolean logic, nesting, and proximity), they automatically queried Yahooligans! using this syntax. Similarly, children queried the engine in natural language assuming that this search feature was provided.

Children looped searches and hyperlinks more frequently than graduate students did. What may explain frequent looping is memory load and low recall associated with using the Web (Cockburn \& Jones, 1996). Since children possess lower memory recall than adults (Siegler, 1991), they are prone to loop searches and hyperlinks more frequently. Another factor that may have affected frequent looping is the design of the Next Search button that appeared at the bottom of the screen when results were returned. There are no instructions on how to use this feature from the retrieval interface. When a child experiences cognitive overload, a child may click on the button often hoping to find new results.

Like prior research, this study revealed that both children and adults used Netscape Back command to navigate among Web pages. In fact, frequent use of this command seems to be common among Web users, regardless of age. The study by Large et al. (1999), for example, shows that backtracking accounted for $90 \%$ of all children's Web activities. Catledge and Pitkow's research findings reveal that $41 \%$ of the browsing patterns of users in their study accounted for activating the Back button (cf. Catledge \& Pitkow, 1995). Tauscher and Greenberg (1997) found that while half of graduate students' navigation actions were open URL addresses, $30 \%$ included the Back button. Consequently, backtracking by children and graduate students in this study is considered typical of Web users. However, this behavior raises the issue of efficiency in using the Web. What happens when a user retrieves Web pages from sites that do not allow going Back?

Although "age" did not seem to be a factor that influenced the two groups' informationseeking behaviors, it did have an impact on their information needs.

\subsection{Children's perceptions}

Most children liked using Yahooligans! mainly because it provides keyword searching, has colorful graphics, is easy and fun to use, and is part of the Web. They, however, were distressed about its slow response time, lack of relevant information, and the zero hits it returns. Few students found the screen display confusing, especially the structure of subject categories and sites within categories. Children made these recommendations for improving Yahooligans!: 
Add more sites, which denotes that the size of the database should be enhanced; Add more categories, which implies that information organization and representation should be strengthened: add more keywords, which indicates that the indexing mechanism should be enriched; Improve screen display, which refers to the structure of the retrieval interface that includes subject categories and sites within categories; Improve response time, which denotes that Web pages should load much faster.

\subsection{Graduate students' perceptions}

Graduate students liked Yahooligans!' hierarchical structure due to its simplicity and comprehensiveness. They also favored the simplicity of the engine's design, especially its uncluttered screen and colorful graphics. Like the children, however, they were dissatisfied with the zero hits it returns. As one student noted: It returns no results instead of returning possible results. This student expected that, instead of zero hits, the engine should recommend "alternative" solutions for improving search strategies.

Graduate students made more sophisticated recommendations for improving Yahooligans!: (a) provide a more in-depth database with more sites; (b) improve the help files, make headings and text in the help files more precise, and include how to use Boolean in these files; (c) employ Boolean operators; (d) add a browsable index; (e) add more search options (phrases, proximity, and nesting); (f) improve keyword searching; (g) enhance response time; (h) and reduce the number of redundant sites.

System developers describe in the online Help file that they want Yahooligans! to be "the websurfing tool of choice for children, parents, and teachers" (Yahooligans!, 1994-2000). To make this engine/directory the "tool of choice", however, system designers should re-evaluate the engine's search capabilities, performance effectiveness, and design interfaces vis-à-vis the information needs and behaviors of both children and adults.

\section{Implications}

The findings of this study have implications for user training and system design improvement.

\subsection{Web training}

Web training adds a new dimension to information skills. Children need to adapt to the use of the Web and search engines to learn new techniques that support effective navigation (Bilal, 2000). In this study, children and graduate students were bound to a limited Web space (as opposed to the whole Web) to find a fact-based task that had a target answer. Fifty percent of the children failed in finding the correct answer. As many school curricula include use of the Web for classrelated assignments, school librarians and teachers need to provide effective Web training programs. In 1986, Mancall, Aaron, and Walker suggested that information skills programs "must consider levels of cognitive development and, as importantly, pay attention to the process skills students need to plan and evaluate all aspects of information utilization and retrieval" (p. 23). Today, these skills are vital for using the Web effectively. Students should be able to assess their 
own knowledge state and understanding of the task, plan appropriate search strategies, and develop techniques to evaluate the steps in their information-seeking process (Mancall, Aaron, \& Walker, 1986). They should also be able to adjust their personal mental state to how information is organized and structured in the search engine they use.

Providing effective Web training requires measuring and mapping users' thoughts, actions, and affective behaviors (Nahl, 1998). This calls upon information professionals to familiarize themselves with a user's cognitive style, habits, and feelings so that they develop a better understanding of a user's information seeking process and, subsequently, provide the affective support and guidance needed throughout the process.

\subsection{System design}

Yahooligans! is known as a directory rather than a search engine. It allows keyword searching, but its online Help does not contain instructions about how to search the directory, or describe the search syntax supported and not supported. The search instructions under Help are vague and misleading. They read: "Yahooligans! will search to find listings in our database that contain all of the search words" (Yahooligans!, 1994-2000). In addition, the zero hits Yahooligans! returns is mainly due to its poor structure of the so-called keyword searching. As described in the Help file, the engine does not search for keywords in text of sites; rather it relies on the "placement of sites in appropriate categories and descriptive comments". Since this mechanism is embedded in Yahooligans!' design, user problems could still be minimized by providing intelligent user interfaces and software agents to assist users during the search process.

Yahooligans! has sites for teachers and parents. However, it does not allow for advanced search syntax. It is recommended that Yahooligans!'s designers employ two interfaces: one simple and one advanced. Users who possess advanced search skills will definitely benefit from using advanced syntax.

Like prior research (Kafai \& Bates, 1997; Large et al., 1999; Schacter et al., 1998; Wallace \& Kupperman, 1997), the findings of this study reveal that many children apply natural language in querying search engines. This suggests the need for a natural language interface or an intelligent interface that translates a user's query into the language employed in the system.

\section{Conclusions}

This study reported the results of children's and graduate students' information-seeking behaviors and success in using Yahooligans! to find the answer to a fact-finding task. It analyzed the cognitive, physical, and affective behaviors of 14 seventh-grade science children and nine graduate students in information science. It applied Bilal's Web Traversal Measure that quantified the two groups' effectiveness and efficiency in using Yahooligans!, as well as quality of Web moves they made (cf. Bilal, 2000).

Although graduate students were more successful in finding the target answer to the search task, and were more effective and efficient in using Yahooligans!, they shared common information-seeking behavior with the children. The major factors that contributed to the differences in information seeking between the two groups were: (a) ability to recover from "breakdowns", 
(b) navigational style, and (c) focus on task. The fact that graduate students used a search engine with a simple interface may have added to their higher performance.

Examination of children's and graduate students' information seeking from the cognitive, physical, and affective perspectives provided insights into their traversal activities in using Yahooligans! It also added to our understanding of the vitality affective states play in the information seeking process.

Use of the Web Traversal Measure proved to be effective for fact-finding tasks. This measure quantified effectiveness, efficiency, and quality of Web moves. Its usability for open-ended tasks, however, remains to be proven.

The fact that both children and graduate students experienced cognitive difficulties in using Yahooligans! suggests the need for effective Web training. Both student groups had inadequate knowledge of how to use the search engine. With effective training, these users may be able to adjust their existing knowledge to the rules provided in various search engines. Children, per se, need to learn how to scan, analyze, evaluate, extract, and synthesize information on the Web. These skills are vital for their information literacy and are at the heart of Information Power (1998) and Big6 Skills of information problem solving (Eisenberg \& Berkowitz, 1990).

This study revealed that Yahooligans! had several limitations that affected children and graduate students' information seeking. System designers should improve the structure of keyword searching and provide intelligent interfaces that support children and adults' information seeking.

More research is needed on the understanding of children's and adults' information seeking behaviors on the Web. A comparison of these users' behaviors in navigating more than one search engine to find information on a variety of search tasks will provide more insights into the significant differences that exist between these two groups. Use of "normal" adults rather than graduate students in information science will establish the baseline necessary to design or adjust search interfaces to support the information-seeking behavior of different users.

\section{Appendix A}

Example of application of the Web Traversal Measure ${ }^{a}$

\begin{tabular}{llll}
\hline TMs & Description & SAs & WSA $^{\mathrm{b}}$ \\
\hline 1 & Click on Science and Nature & 1 & 1 \\
1 & Type alligators & 1 & 1 \\
1 & Scroll screen & - & - \\
1 & Click on Science and Nature: Animals: & 1 & 1 \\
& Reptiles: Alligators and Crocodiles & \multicolumn{2}{|}{} \\
1 & Move mouse over links & - & - \\
1 & Scroll screen & - & - \\
1 & Click on Crocodilian Species: Chinese & 1 & 0.5 \\
& Alligator & \multicolumn{2}{|}{} \\
1 & Scroll screen & - & - \\
1 & Move mouse over links & - & - \\
1 & Click on Net Search button & - & -
\end{tabular}




\begin{tabular}{|c|c|c|c|}
\hline 1 & Click on Stop button & - & - \\
\hline 1 & Click on Back button & - & - \\
\hline 1 & Click on Zoos & 1 & 0.5 \\
\hline 1 & $\begin{array}{l}\text { Click on Science and Nature: Animals: } \\
\text { Reptiles: Alligators and Crocodiles }\end{array}$ & 1 & 1 \\
\hline 1 & Type alligator & 1 & 1 \\
\hline 1 & Scroll screen & - & - \\
\hline 1 & Move mouse over links & - & - \\
\hline 1 & Type animals & 1 & 0.5 \\
\hline 1 & Click on Back button & & \\
\hline 1 & Type alligators & 1 & 1 \\
\hline 1 & Click on Gator Hole (target hyperlink) & 1 & 1 \\
\hline 1 & Scroll screen & - & - \\
\hline 1 & Highlight text for answer & - & - \\
\hline \multicolumn{4}{|c|}{ Total } \\
\hline 23 & & 10 & 8.5 \\
\hline
\end{tabular}

Weighted efficiency score:

$$
\sum_{i=1}^{10} \mathrm{WSA}_{i} / \sum_{j=1}^{23} \mathrm{TM}_{i}=8.5 / 23=36.9 \%
$$

Quality moves:

$$
\sum_{i=1}^{10} \mathrm{SA}_{i} / \sum_{i=1}^{23} \mathrm{TM}_{i}=10 / 23=43.5 \%
$$

$\mathrm{TM}=$ Transcribed Move; $\mathrm{SA}=$ Selection Action; WSA = Weight for each SA.

${ }^{\mathrm{a}}$ This measure is based on Bilal (2000) Web Traversal Measure.

${ }^{\mathrm{b}}(1=$ relevant $) ;(0.5=$ semi-relevant $)$. Note: Each TM is a move; each SA is a "meaningful" TM.

\section{References}

AASL \& AECT (1998). Information power: Building partnerships for learning. Chicago, IL: American Library Association.

Bilal, D. (1998). Children's search processes in using World Wide Web search engines: an exploratory study. In Proceedings of the 61st ASIS annual meeting, 35, October 24-29, 1998, Pittsburgh, PA (pp. 45-53).

Bilal, D. (1999). Web search engines for children: a comparative study and performance evaluation of Yahooligans!, Ask Jeeves for Kids ${ }^{\mathrm{TM}}$, and Super Snooper ${ }^{\mathrm{TM}}$. In Proceedings of the 62nd ASIS annual meeting, October 31-November 4, 1999, Washington, DC (pp. 70-82).

Bilal, D. (2000). Children's use of the Yahooligans! Web search engine. I. Cognitive, physical, and affective behaviors on fact-based tasks. Journal of the American Society for Information Science, 51(7), 646-665. 
Bilal, D. (2001). Children's use of the Yahooligans! Web search engine. II. Cognitive and physical behaviors on research tasks. Journal of the American Society for Information Science, 52(2), 118-137.

Bilal, D., \& Watson, J. S. (1998). Children's paperless projects: inspiring research via the Web. In Proceedings of the 64th IFLA general conference, August 16-21, 1998, Amsterdam, The Netherlands (Booklet 3) (pp. 101-107). Available: http://www.ifla.org/IV/ifla64/009-13e.htm.

Catledge, L. D., \& Pitkow, J. E. (1995). Characterizing browsing strategies in the World-Wide Web. Available: http:// www.igd.fhg.de/www/www95/proceedings/papers/80/userpatterns/UserPatterns.Paper4.formatted.html. Accessed on January 15, 2000.

Chen, C., Czerwinski, M., \& Macredie, R. (2000). Individual differences in virtual environments: introduction and overview. Journal of the American Society for Information Science, 51(6), 499-507.

Choo, C. W., Detlor, B., \& Turnbull, D. (1999). Information seeking on the Web - an integrated model of browsing and searching. In Proceedings of the 62nd ASIS annual meeting, 36, October 31-November 4, 1999, Washington, DC (pp. 3-16).

Cockburn, A., \& Jones, S. (1996). Which way now? Analyzing and easing inadequacies in WWW navigation. International Journal of Human-Computer Studies, 45, 105-129.

Dervin, B. (1983). Information as a user construct: the relevance of perceived information needs to synthesis and interpretation. In S. A. Ward, \& L. A. Reed (Eds.), Knowledge structure and use: Implications for synthesis and interpretation. Philadelphia, PA: Temple University Press.

Eisenberg, M. B., \& Berkowitz, R. E. (1990). Information problem-solving: The big six skills approach to library and information skills instruction. Norwood, NJ: Ablex Publishing.

Ellis, D. (1989). A behavioural model for information retrieval system design. Journal of Information Science, 15(4/5), 237-247.

Ellis, D., Cox, D., \& Hall, K. (1993). A comparison of the information seeking patterns of researchers in the physical and social sciences. Journal of Documentation, 49(4), 356-369.

Ellis, D., \& Haugan, M. (1997). Modelling information seeking patterns of engineers and research scientists in an industrial environment. Journal of Documentation, 53(4), 384-403.

Fidel, R. et al. (1999). A visit to the information mall: Web searching behavior of high school students. Journal of the American Society for Information Science, 50(1), 24-38.

Ingwersen, P. (1982). Search procedures in libraries analyzed from the cognitive point of view. Journal of Documentation, 38(3), 165-191.

Ingwersen, P. (1992). Information retrieval interaction. London: Taylor Graham.

Ingwersen, P. (1996). Cognitive perspectives of information retrieval interaction: elements of a cognitive IR theory. Journal of Documentation, 51(1), 3-50.

Jansen, M. J., Spink, A., \& Saracevic, T. (2000). Real life, real users, real needs: a study of analysis of user queries on the Web. Information Processing and Management, 36(2), 207-227.

Kafai, Y., \& Bates, M. J. (1997). Internet web-searching instruction in the elementary classroom: building a foundation for information literacy. School Library Media Quarterly, 25(2), 103-111.

Kuhlthau, C. C. (1993). Seeking meaning: A process approach to library and information services. Norwood: Ablex Publishing.

Large, A., \& Beheshti, J. (2000). The Web as a classroom resource: reactions from the users. Journal of the American Society for Information Science, 51(12), 1069-1080.

Large, A., Beheshti, J., \& Moukad, H. (1999). Information seeking on the Web: navigational skills of grade-six primary school students. In Proceedings of the 62nd ASIS annual meeting, October 31-November 4, 1999, Washington, DC (pp. 84-97).

Lazonder, A. W., Biemans, J. A., \& Wopereis, G. J. H. (2000). Differences between novice and experienced users in searching information on the World Wide Web. Journal of the American Society for Information Science, 51(6), 576-581.

Mancall, J. C., Aaron, S. L., \& Walker, S. A. (1986). Educating students to think: the role of the school library media program. School Library Media Quarterly, 15(Fall), 18-27.

Marchionini, G. (1995). Information seeking in electronic environments. Cambridge, UK: Cambridge University.

Meghabghab, D. B. (1995). CD-ROM vs. online vs. Internet: search strategies and evaluation from the user's perspective. In Proceedings of the 16th national online meeting, May 2-4, 1995, New York (pp. 295-307). 
Nahl, D. (1997). Information counseling inventory of affective and cognitive reactions while learning the Internet. Internet Reference Services Quarterly, 2(2/3), 11-33.

Nahl, D. (1998). Learning the Internet and the structure of information behavior. Journal of the American Society for Information Science, 49(11), 1017-1023.

Nahl, D., \& Meer, P. (1997). User-centered assessment of two web browsers: errors, perceived self-efficacy, and success. In Proceedings of the 60th ASIS annual meeting, November 1-6, 1997, Washington, DC (pp. 89-97).

Palmquist, R. A., \& Kim, K. S. (2000). Cognitive style and on-line database search experience as predictors of Web search performance. Journal of the American Society for Information Science, 51(6), 558-566.

Qiu, L. (1994). Frequency distributions of hypertext path patterns: a pragmatic approach. Information Processing and Management, 30(1), 131-140.

Saracevic, T. (1997). Users lost: reflections on the past, future, and limits of information science. SIGIR Forum, 31(2), $16-27$.

Schacter, J., Chung, G. K. W. K., \& Dorr, A. (1998). Children's Internet searching on complex problems: performance and process analyses. Journal of the American Society for Information Science, 49(9), 840-849.

Siegler, R. S. (1991). Children's thinking. Englewood Cliffs, NJ: Prentice-Hall.

Tauscher, L., \& Greenberg, S. (1997). How people revisit web pages: empirical findings and implications for the design of history systems. International Journal of Human-Computer Studies, 47, 97-137.

Vakkari, P. (1999). Task complexity, problem structure and actions: integrating studies on information seeking and retrieval. Information Processing and Management, 35, 819-837.

Wallace, R. \& Kupperman, J. (1997). Online search in the science classroom: benefits and possibilities. Available: http:// mydl.soe.unich.edu/papers/online/search.pdf. Accessed on April 2, 1999.

Wang, P., Hawk, W. B., \& Tenopir, C. (2000). Users' interaction with the World Wide Web resources: an exploratory study using a holistic approach. Information Processing and Management, 36(2), 229-251.

Wilson, T. D. (2000). Human information behavior. Information Science Research, 3(2), 49-56.

Wolfram, D., \& Ross, N. C. M. (2000). End user searching on the Internet: an analysis of term pair topics submitted to the Excite search engine. Journal of the American Society for Information Science, 51(10), 949-958.

Yahooligans! (1994-2000). Available: http//www.yahooligans.com. Accessed on April 15, 1999 and February 7, 2000. 